\title{
The Interday Reliability of Leg and Ankle Musculotendinous Stiffness Measures
}

\author{
Ken A. McLachlan, Aron J. Murphy, Mark L. Watsford, and Sven Rees \\ University of Technology, Sydney
}

\begin{abstract}
Two popular methods of assessing lower body musculotendinous stiffness include the hopping and oscillation tests. The disparity and paucity of reliability data prompted this investigation into leg musculotendinous stiffness $\left(\mathrm{K}_{\operatorname{leg}}\right)$ and ankle musculotendinous stiffness $\left(\mathrm{K}_{\text {ank }}\right)$ measures. $\mathrm{K}_{\text {leg }}$ and $\mathrm{K}_{\text {ank }}$ were assessed on three separate occasions in 20 female subjects. $\mathrm{K}_{\mathrm{leg}}$ was determined using bilateral hopping procedures conducted at $2.2 \mathrm{~Hz}$ and $3.2 \mathrm{~Hz}$ frequencies. $\mathrm{K}_{\text {ank }}$ was assessed by perturbation of the subject's ankle musculotendinous unit on an instrumented calf raise apparatus at $70 \%$ of maximum isometric force (MIF). Excellent reliability was produced for all $\mathrm{K}_{\mathrm{leg}}$ measures between all days, whereas $\mathrm{K}_{\text {ank }}$ exhibited acceptable reliability after one session of familiarization. No relationship was evident between $\mathrm{K}_{\operatorname{leg}}$ and $\mathrm{K}_{\text {ank }}$. It was concluded that no familiarization session was required for $\mathrm{K}_{\text {leg }}$ at the test frequencies and conditions tested, whereas at least one familiarization session was needed to ensure the reliable assessment of $\mathrm{K}_{\mathrm{ank}}$.
\end{abstract}

Key Words: leg stiffness, maximal isometric force, ankle stiffness

The lower body musculotendinous unit, consisting of contractile muscle and the attached tendinous structures, is frequently utilized to determine the

The authors are with the Biomechanics Laboratory in the School of Leisure, Sport, and Tourism; University of Technology, Sydney; Lindfield NSW 2070, Australia. body's ability to store and utilize rebound energy (Bobbert et al., 1996). This rebound energy has previously been shown to originate from the storage and restitution of elastic strain energy (Asmussen \& Bonde-Petersen, 1974; Komi \& Bosco, 1978; Shorten, 1987). However, other research suggests that this energy is used in combination with enhanced muscular activation and neural stretchreflex mechanisms (Dietz et al., 1979; Sinkjaer et al., 1998) to augment stretch-shortening cycle movement performances such as running and jumping. Properties of this structure are trainable (Spurrs et al., 2003; Wilson et al., 1992) with the stiffness/compliance potentially influencing the efficiency and performance of a human bounding system through the storage and utilization of rebound energy.

Stiffness is defined as the property of a system to resist an applied change in length (Kuitunen et al., 2002). Measurement of lower body stiffness is commonly determined by either the assessment of the entire lower leg $\left(\mathrm{K}_{\mathrm{leg}}\right)$ in a functional bilateral hopping test protocol or by the isolated assessment of ankle stiffness $\left(\mathrm{K}_{\text {ank }}\right)$ using the oscillation technique. Both measures are based on the premise that the musculotendinous unit can independently or collectively be modeled as a damped linear springmass system (Shorten, 1987).

A wide range of $\mathrm{K}_{\mathrm{leg}}$ values (13.9 to 112.7 $\mathrm{kN} \cdot \mathrm{m}^{-1}$ ) have been quantified in previous research (Bret et al., 2002; Chelly \& Denis, 2001; Farley et al., 1991, 1998; Farley \& Morgenroth, 1999; Ferris $\&$ Farley, 1997). Given the functional nature of this test, $\mathrm{K}_{\mathrm{leg}}$ determination methods depict the mechan- 
ics of general running and locomotion extremely well (Blickhan, 1989; McMahon \& Cheng, 1990). A relationship between $\mathrm{K}_{\mathrm{leg}}$ and running performance has been previously established (Arampatzis et al., 1999; Bret et al., 2002; Chelly \& Denis, 2001). Gunther and Blickhan (2002) took this applied concept further by examining the characteristics of global and local joint level leg stiffness (including $\mathrm{K}_{\text {ank }}$ ) adjustment during running performance and identified a optimum $\mathrm{K}_{\text {ank }}$ to knee stiffness ratio that varies with velocity to elicit performance and ensures leg stability. Other research has confirmed the existence of a $\mathrm{K}_{\mathrm{leg}}$ segmental ratio but the degree of contribution varies considerably between studies (Arampatzis et al., 1999; Farley \& Morgenroth, 1999; Kuitunen et al., 2002). To the authors' knowledge, the reliability of $\mathrm{K}_{\mathrm{leg}}$ methods using the hopping technique remains undetermined.

Similarly, a wide range of absolute $\mathrm{K}_{\text {ank }}$ values ( 3.3 to $45.9 \mathrm{kN} \cdot \mathrm{m}^{-1}$ ) have been quantified in the literature using subjects of both genders and/or varied training status (Hunter \& Spriggs, 2000; McNair \& Stanley, 1996; Murphy et al., 2003; Spurrs et al., 2003; Watsford et al., 2003). The ankle joint is isolated during a typical $\mathrm{K}_{\mathrm{ank}}$ assessment allowing stiffness of the triceps surae to be examined. This measure has been implicated as a significant contributor to overall $\mathrm{K}_{\mathrm{leg}}$ (Farley \& Morgenroth, 1999) and running performance (Stefanyshyn \& Nigg, 1998) and may play a role in the incidence of lower leg injuries (Butler et al., 2003). The reliability of such a test has mixed results of between $r=.68$ and .95 for unilateral measures based on 30-90\% of maximum isometric force (MIF) (Hunter \& Spriggs, 2000; Murphy et al., 2003).

The assessment of $\mathrm{K}_{\text {leg }}$ and $\mathrm{K}_{\text {ank }}$ are clearly popular methods of measuring stiffness of the lower body. However, the variety of methodologies adopted by previous researchers has resulted in varying levels of reliability for $\mathrm{K}_{\mathrm{ank}}$, whereas no reliability data exists for $\mathrm{K}_{\operatorname{leg}}$ measures. Therefore, the current study attempted to address these issues by assessing the reliability and relationship of both measures.

\section{Methods}

Twenty healthy females (age $=20.7 \pm 3.2$ years, mass $=61.8 \pm 7.6 \mathrm{~kg}$, height $=166.02 \pm 6.1 \mathrm{~cm}$ ) from a variety of sporting backgrounds provided their written consent to participate in this study.
Subjects were required to be regularly involved in physical activity while not possessing any recent (within 1 year) or previously significant lower body injury. The Human Research Ethics Committee of the University of Technology, Sydney, approved the procedures.

Subjects undertook three sessions of testing that were completed over a maximum period of 21 days and were instructed to abstain from completing any form of lower body training 24 hours prior to each testing session. A standardized warm up and cool down consisting of cycling, submaximal running, and stretching with a focus on the lower body was completed before and after stiffness assessment sessions. Each stiffness test was conducted 3 to 7 days apart to avoid any effects from previous testing sessions. All tests were conducted in the Biomechanics Laboratory of the University of Technology, Sydney.

$\mathrm{K}_{\text {leg }}$ assessment was conducted on a force platform (Onspot, Wollongong, Australia) using various hopping tests. The force platform was leveled and calibrated to a series of known masses before use. Subjects were instructed to hop bilaterally at movement frequencies of $2.2 \mathrm{~Hz}$ and $3.2 \mathrm{~Hz}$ normally and for maximum height (Farley et al., 1991) in time with a digital metronome (Seiko, Tokyo, Japan). The various hopping speeds and conditions were incorporated into the test protocol to examine the reliability of the spring-mass system at cyclic bounding rates and eccentric loading forces more comparable with dynamic movements such as high-speed running and jumping, where additional muscle recruitment is involved. Throughout each trial, subjects were barefoot and were instructed to keep their hands on their hips to negate any upper body contribution to each hop. Once steady-state hopping and synchronization with the metronome was achieved, data from the force platform was sampled for $5 \mathrm{~s}$ at $1,000 \mathrm{~Hz}$ and recorded in computer. Trials at both frequencies and hopping heights were accepted when three hops within the 5 -s hopping force profile were within $\pm 2 \%$ of the designated movement frequency (Farley et al., 1998; Ferris \& Farley, 1997). As a consequence, repeated trials for this measure were rarely required and were estimated at no more than $2 \%$ of the time for each test occasion.

A spring-mass system consists of a point mass (body mass) and a single linear spring (leg spring) 
(Blickhan, 1989), with the linear spring representing the overall stiffness of the multijointed leg. As the hopping tests were conducted bilaterally, $\mathrm{K}_{\mathrm{leg}}$ was determined as the sum of the stiffness of both legs (Farley et al., 1998). When a subject hopped on the force platform, $\mathrm{K}_{\text {leg }}$ was calculated as the ratio of the force/length change of the "internal leg spring" occurring from the start to the middle of the ground contact phase where the leg spring was maximally compressed, shown mathematically by

$$
K_{\text {leg }}=\frac{F_{\text {peak }}}{\Delta L}
$$

where $F_{\text {peak }}=$ maximum ground reaction force and $\Delta L=$ maximum leg spring displacement change.

$\Delta \mathrm{L}$ was calculated by double integration of the vertical acceleration of the center of mass as calculated from the vertical ground reaction force (Cavagna, 1975). Three consecutive hops were considered to provide an average $\mathrm{K}_{\mathrm{leg}}$ value.

MIF assessment determined the percentage of maximum loading to use during subsequent $\mathrm{K}_{\text {ank }}$ oscillation tests. MIF was measured bilaterally with the subject performing a seated muscular contraction on an instrumented calf raise apparatus (Figure 1) (Onspot, Wollongong, Australia). Force data was collected via a strain gauge (Celtron, California, USA) secured in series with a rigid foot platform. The strain gauge was calibrated with a series of known masses before use. The apparatus contained an adjustable winch that secured the swing arm of the machine in place to an accuracy of $.25^{\circ}$, ensuring that a purely static contraction of the lower limb took place. Subjects sat in the machine barefoot, with their hips, knees, and ankles positioned at $90^{\circ}$. The thigh pad, constructed of high-density foam to minimize damping of the force response, was positioned to ensure the relative segment position between subjects was the same regardless of limb length. All machine and position settings were recorded to ensure accurate repeatability of all tests for each subject. Upon the experimenter's signal, subjects contracted the triceps surae, producing maximum force against the thigh pad "as hard and as fast as possible" for $3 \mathrm{~s}$. Data were amplified, sampled at 1,000 Hz, and recorded by computer. MIF was determined as the peak of the force produced during this time. A rest period of 2 min was implemented between trials to ensure the application of maximum effort, with the average of two trials taken as the MIF value.
The same instrumented calf raise apparatus used for the MIF test (Figure 1) was then utilized to determine $\mathrm{K}_{\mathrm{ank}}$ by way of an oscillation technique commonly used in previous research settings (McNair \& Stanley, 1996; Walshe \& Wilson, 1997; Walshe et al., 1996). This method permitted the isolation of the triceps surae musculotendinous unit for analysis.

With the swing arm tension removed through the release of winch pressure, subjects assumed a position identical to that of the MIF test. However, a load of $70 \%$ of MIF was applied through the pad resting on the distal thigh of the subject to calculate $\mathrm{K}_{\text {ank }}$. This loading was chosen as it has been previously reported in the literature to produce maximum stiffness values (Sinkjaer et al., 1998; Walshe et al., 1996). While maintaining the $90^{\circ}$ ankle position and with the $70 \%$ MIF load resting on the subject's distal thighs, a brief perturbation in the order of 100-200 N was applied by the experimenter's hands in a vertical direction through the thigh pad and consequently on the subject's lower legs. As the experimenter performed the perturbations manually, variations in the magnitudes were expected. However, a system will independently oscillate at a natural frequency regardless of the magnitude of the applied perturbation (Shorten, 1987; Walshe et al., 1996). The subject was instructed to maintain a constant level of

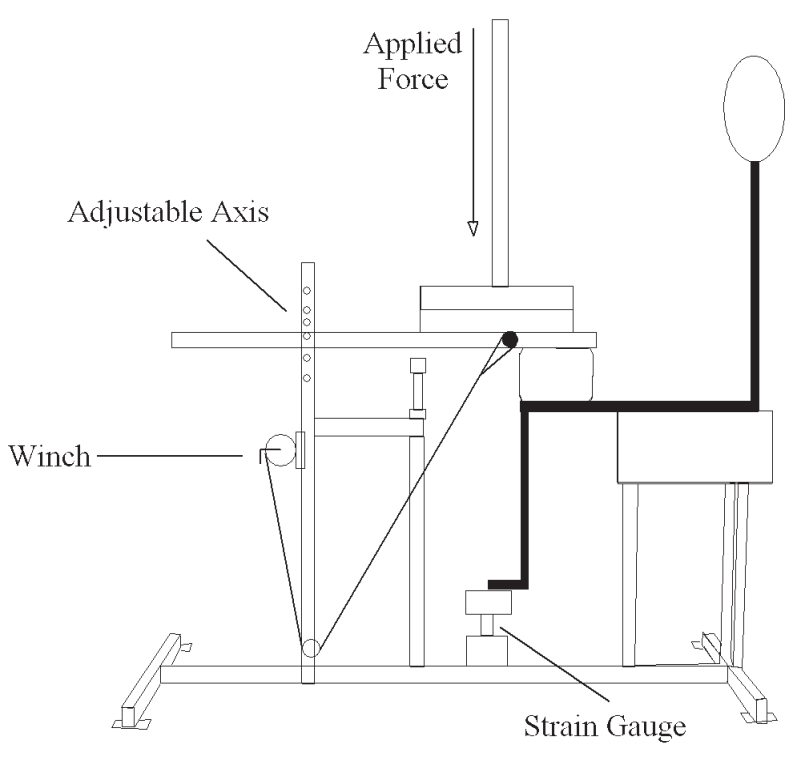

Figure 1 - Schematic representation of the instrumented apparatus used for maximal isometric force and musculotendinous stiffness assessment. 
muscular contraction and not to intervene throughout the testing procedures, particularly in response to the applied perturbation. The high-density nature of the thigh pad ensured minimal damping of the oscillatory response. Analysis of the force curves and evidence of minimal noise in the trace following each trial ensured that the oscillations during the trials were indicative of natural resonance only (Figure 2). If the oscillatory force profile exhibited any sign of excessive noise or subject intervention, the trial was repeated. It was estimated that this occurred $10 \%$ of the time on each testing occasion. Two oscillation trials were completed and averaged for each subject.

The analogue force oscillations that resulted were amplified, digitally sampled $(1,000 \mathrm{~Hz})$, and stored on computer. The data were then fed into a low-pass 4th-order Butterworth filter with a cut-off frequency of $12 \mathrm{~Hz}$ before the calculation of stiffness measurements took place. The damped free oscillations that resulted from perturbation of the ankle musculotendinous unit were modeled via the following 2 nd-order linear equation:

$$
m a+c v+K x=m g
$$

where $m=$ mass of the system $(\mathrm{kg}), a=$ acceleration of the system $\left(\mathrm{m} \cdot \mathrm{s}^{-2}\right), c=$ damping coefficient $\left(\mathrm{Ns} \cdot \mathrm{m}^{-1}\right), v=$ velocity of the system $\left(\mathrm{m} \cdot \mathrm{s}^{-1}\right), k=$ stiffness of the system $\left(\mathrm{kN} \cdot \mathrm{m}^{-1}\right), x=$ displacement of the system (m), and $g=$ acceleration due to gravity $\left(\mathrm{m} \cdot \mathrm{s}^{-2}\right)$.

The stiffness $(K)$ of the system was determined using the equation

$$
K=4 m f^{2} \pi^{2}+\frac{c^{2}}{4 m}
$$

where $m=$ mass of the system $(\mathrm{kg}), f=$ damped oscillatory frequency of the musculotendinous unit, and $c=$ damping coefficient $\left(\mathrm{Ns} \cdot \mathrm{m}^{-1}\right)$.

The damped oscillatory frequency $(f)$ was quantified as the inverse of the period between successive oscillatory force peaks (Figure 2). Two successive oscillatory peaks and depressions were included in the sinusoidal curve analysis ensuring that the latency in the peaks did not exceed $80 \mathrm{~ms}$ (Walshe et al., 1996). By calculating the damping ratio or the natural logarithmic decrement of the first two successive peak forces and obtaining the gradient of the line (s), the damping coefficient (c) was determined:

$$
c=4 \pi m f_{n} s
$$

where the natural frequency, $f_{n}$, of the system is expressed as

$$
f_{n}=\left[\frac{f^{2}}{\left(1-s^{2}\right)}\right]^{\frac{1}{2}}
$$

and the damping ratio $s$ is calculated by

$$
\ln \left(F_{1} / F_{2}\right)
$$

where $F_{1}=$ Peak Force 1 and $F_{2}=$ Peak Force 2 .

All statistical data was analyzed using the Statistical Package for Social Science (SPSS, version 11.0). The requirements for applying an ANOVA to the data series were verified using statistical procedures in SPSS. Differences between subject group

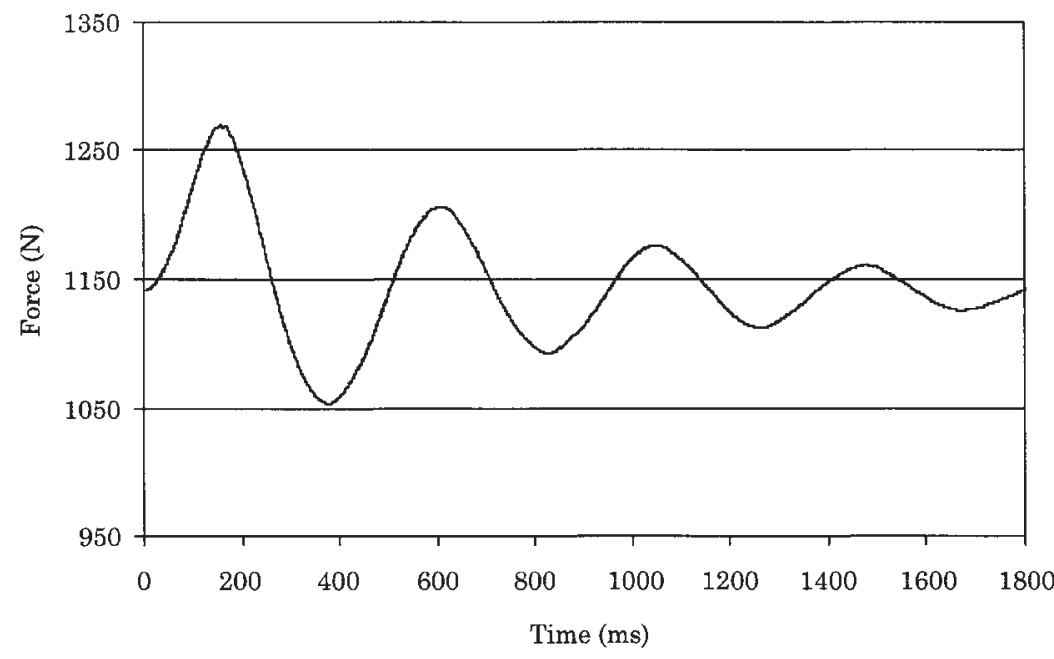

Figure 2 - Schematic representation of the damped force oscillations from perturbation of a loaded triceps surae musculotendinous unit. 
means for each test occasion were assessed using simultaneous pairwise comparisons in ANOVA. In addition, the interday reliability of the various stiffness data was assessed by way of intraclass correlations (ICCs) derived from repeated-measures ANOVA. Further interindividual variance was examined by calculating the differences between Pearson's correlation coefficients using the $t$ test formula developed by Hotelling (1940). The method error (ME), calculated as a coefficient of variation percentage, was determined to examine intraindividual differences, in line with the procedures outlined by Sale (1991). The effect size (ES) was also calculated to quantify the magnitude of any stiffness trends. Finally, Pearson's correlation coefficients were used to assess the degree of relationship between $\mathrm{K}_{\operatorname{leg}}$ and $\mathrm{K}_{\text {ank }}$. Statistical significance for all procedures was accepted at an alpha level of $p<.05$.

\section{Results}

Values for $\mathrm{K}_{\text {leg }}$, MIF, and $\mathrm{K}_{\mathrm{ank}}$ across the 3 days of assessment are presented in Table $1 . \mathrm{K}_{\mathrm{leg}}$ values increased as the frequency and height of the hopping test increased; however, no significant differences were evident for any of the $\mathrm{K}_{\text {leg }}$ tests across the days of testing. MIF values increased significantly by $7.9 \%$ from Days 1 to $2(p<.05)$ and nonsignificantly by $2.8 \%$ from Days 2 to $3 . \mathrm{K}_{\mathrm{ank}}$ values significantly increased by $12.2 \%$ between Days 1 and $2(p<.05)$ and by $2.8 \%$ between Days 2 and $3(p<.05)$.
$\mathrm{K}_{\text {leg }}$ reliability results (Table 2) produced excellent levels at all levels and test modalities with ICCs ranging from $r=.85-.95(p<.01)$. Hotelling's $t$ test of correlation coefficients revealed no significant differences between any of the $\mathrm{K}_{\mathrm{leg}}$ correlation coefficients (Days 1-2 vs. Days 2-3) with values in the range of -.4 to .32 . The $\mathrm{ME}$ for $\mathrm{K}_{\text {leg }}$ was low, ranging between $2.78 \%$ and $4.98 \%$, whereas the ES was small across most $\mathrm{K}_{\text {leg }}$ and $\mathrm{K}_{\text {ank }}$ test variants.

MIF is an integral component of the methodology for $\mathrm{K}_{\mathrm{ank}}$ assessment (in determining an appropriate load for the oscillation test); therefore, the reliability of this data was considered important. The ICC for MIF (Table 2) produced a moderate coefficient of $r=.58(p<.01)$ between Days 1 and 2 , which improved to $r=.86(p<.01)$ between Days 2 and 3. According to Hotelling's $t$-test value of -3.12 the MIF correlation coefficients between test occasions were significantly different $(p<.05)$. ME results for MIF improved from $8.71 \%$ to $5.81 \%$ as was done with ES. Similar to MIF results, $\mathrm{K}_{\text {ank }}$ ICCs were initially poor between Days 1 and $2(r$ $=.43 ; p<.05)$ but improved to excellent $(r=.93$, $p<.01)$ between Days 2 and 3. Hotelling's $t$ test value of $-5.89(p<.05)$ indicated the existence of significant differences between $\mathrm{K}_{\mathrm{ank}}$ correlation coefficients for Days 1 and 2 compared with Days 2 and $3(p<.05)$. ME results for $\mathrm{K}_{\text {ank }}$ tests were moderate at $9.57 \%$ when comparing Days 1 and 2 but improved considerably to $3.15 \%$ when examining the relationship between Days 2 and 3, with the ES also supporting this trend.

Table 1 Musculotendinous Stiffness Values

\begin{tabular}{llll}
\hline Test & Day 1 & Day 2 & Day 3 \\
\hline $\mathrm{K}_{\text {leg }} 2.2 \mathrm{~Hz}$ norm & $16.39 \pm 3.07$ & $16.16 \pm 3.12$ & $16.52 \pm 3.17$ \\
$\mathrm{~K}_{\text {leg }} 2.2 \mathrm{~Hz}$ max & $19.09 \pm 2.61$ & $19.21 \pm 2.82$ & $18.83 \pm 2.38$ \\
$\mathrm{~K}_{\text {leg }} 3.2 \mathrm{~Hz}$ norm & $29.55 \pm 3.16$ & $29.90 \pm 2.89$ & $29.83 \pm 3.15$ \\
$\mathrm{~K}_{\text {leg }} 3.2 \mathrm{~Hz}$ max & $30.59 \pm 3.14$ & $30.70 \pm 2.84$ & $30.37 \pm 3.16$ \\
$\mathrm{MIF}$ & $2.02 \pm .24$ & $2.18 \pm .32^{*}$ & $2.24 \pm .36^{* *}$ \\
$\mathrm{~K}_{\text {ank }}$ & $44.27 \pm 5.70$ & $49.69 \pm 6.19^{* *}$ & $51.06 \pm 5.66^{* * \dagger}$ \\
\hline
\end{tabular}

Note. All units are displayed in means $\pm S D$; musculotendinous stiffness values measured in $\mathrm{kN} \cdot \mathrm{m}^{-1}$; MIF values measured in $\mathrm{kN}$. $\mathrm{K}_{\mathrm{leg}}$ norm: leg musculotendinous stiffness (normal height hopping). $\mathrm{K}_{\mathrm{leg}} \max$ : leg musculotendinous stiffness (maximum height hopping). MIF: maximal isometric force; $\mathrm{K}_{\mathrm{ank}}$ : ankle musculotendinous stiffness.

*Significantly different from the Day 1 value $(p<.05) ; * *$ Significantly different from the Day 1 value $(p<.01)$; †Significantly different from the Day 2 value $(p<.05)$. 
Table 2 Musculotendinous Stiffness Reliability

\begin{tabular}{llllllll}
\hline Test & $\begin{array}{l}\text { Days 1-2 } \\
\text { ICC }\end{array}$ & $\begin{array}{l}\text { ME } \\
(\%)\end{array}$ & ES & $\begin{array}{l}\text { Days 2-3 } \\
\text { ICC }\end{array}$ & $\begin{array}{l}\text { ME } \\
(\%)\end{array}$ & ES & $\begin{array}{l}\text { Hotelling's } \\
t \text { ratio }\end{array}$ \\
\hline $\mathrm{K}_{\text {leg }} 2.2 \mathrm{~Hz}$ norm & $.93^{* *}$ & 4.98 & .10 & $.94^{* *}$ & 4.69 & -.16 & .32 \\
$\mathrm{~K}_{\operatorname{leg}} 2.2 \mathrm{~Hz} \max$ & $.91^{* *}$ & 4.33 & -.06 & $.91^{* *}$ & 4.19 & .34 & -.40 \\
$\mathrm{~K}_{\operatorname{leg}} 3.2 \mathrm{~Hz}$ norm & $.85^{* *}$ & 3.97 & -.17 & $.88^{* *}$ & 3.46 & .03 & -.89 \\
$\mathrm{~K}_{\operatorname{leg}} 3.2 \mathrm{~Hz} \max$ & $.92^{* *}$ & 2.78 & -.05 & $.92^{* *}$ & 2.72 & .16 & -.21 \\
$\mathrm{MIF}$ & $.58^{* *}$ & 8.71 & -.78 & $.86^{* *}$ & 5.81 & -.28 & $-3.12 \dagger$ \\
$\mathrm{K}_{\mathrm{ank}}$ & $.43^{*}$ & 9.57 & -1.29 & $.93^{* *}$ & 3.15 & -.33 & $-5.89 \dagger$ \\
\hline
\end{tabular}

Note. $\mathrm{K}_{\operatorname{leg}}$ norm: leg musculotendinous stiffness (normal height hopping); $\mathrm{K}_{\operatorname{leg}}$ max: leg musculotendinous stiffness (maximum height hopping); MIF: maximal isometric force; $\mathrm{K}_{\text {ank }}$ : ankle musculotendinous stiffness; ICC: intraclass correlation; $r^{2}$ : common variance: ME: method error; ES: effect size.

$*$ Significant intraclass correlation coefficient $(p<.05) ; *$ Significant intraclass correlation coefficient $(p<.01)$; †Significantly different between correlation coefficients $(p<.05)$.

Pearson's product moment correlations between $\mathrm{K}_{\mathrm{leg}}$ and $\mathrm{K}_{\mathrm{ank}}$ were established to determine whether any relationship existed between these measures (Table 3). Given the poor $\mathrm{K}_{\text {ank }}$ reliability for the Days 1-2 measures, data from Day 3 was used for this analysis. Poor correlation coefficients ranging from .07 to .21 indicated the absence of a relationship between $\mathrm{K}_{\text {leg }}$ and $\mathrm{K}_{\mathrm{ank}}$.

\section{Discussion}

The variation and deficiencies in previous reliability research prompted this examination of the interday reliability of $\mathrm{K}_{\mathrm{leg}}$ and $\mathrm{K}_{\mathrm{ank}}$. This study attempted to provide a scientifically established and reliable standard of measuring lower body stiffness to aid in

\section{Table 3 Correlations Between Leg and Ankle} Musculotendinous Stiffness

\begin{tabular}{ll}
\hline $\begin{array}{l}\mathbf{K}_{\text {leg }} \text { Variant } \\
\text { Day 3 }\end{array}$ & $\begin{array}{l}\mathbf{K}_{\text {ank }} \\
\text { Day 3 }\end{array}$ \\
\hline $\mathrm{K}_{\text {leg }} 2.2 \mathrm{~Hz}$ norm & .17 \\
$\mathrm{~K}_{\text {leg }} 2.2 \mathrm{~Hz}$ max & .21 \\
$\mathrm{~K}_{\text {leg }} 3.2 \mathrm{~Hz}$ norm & .13 \\
$\mathrm{~K}_{\text {leg }} 3.2 \mathrm{~Hz} \max$ & .07 \\
\hline
\end{tabular}

Note. $\mathrm{K}_{\mathrm{leg}}$ norm: leg musculotendinous stiffness (normal height hopping); $\mathrm{K}_{\operatorname{leg}}$ max: leg musculotendinous stiffness (maximum height hopping); $\mathrm{K}_{\mathrm{ank}}$ : ankle musculotendinous stiffness. the development of future assessment methodology. Assessment of the reliability of both tests involved the comparison of stiffness data measured across three days.

The $\mathrm{K}_{\operatorname{leg}}$ values in the current study (Table 1) increased as the bounding frequency and height increased, which is in accordance with other research (Farley et al., 1991; Farley \& Morgenroth, 1999; Ferris \& Farley, 1997) suggesting congruence with previous methodology. Very high reliability coefficients for all $\mathrm{K}_{\text {leg }}$ tests were produced in addition to a low ME and a low-to-medium ES. To the authors' knowledge, this study is the first to investigate the repeatability of $\mathrm{K}_{\mathrm{leg}}$ assessment procedures. Retrospectively, the results of this study imply that any $\mathrm{K}_{\mathrm{leg}}$ assessment utilizing the hopping method may be deemed reliable. Stringency was assured in the current study by conducting tests under identical environmental conditions, instructing subjects to hop in time to a digital metronome and by having subjects repeat trials if hops were outside the designated movement frequency by $\pm 2 \%$. These findings also suggest that future $\mathrm{K}_{\mathrm{leg}}$ assessment protocols do not require familiarization sessions and similar methodological procedures should be incorporated in future research to ensure the adequate reliability of $\mathrm{K}_{\mathrm{leg}}$ measures.

A major finding of this study was the achievement of adequate $\mathrm{K}_{\mathrm{ank}}$ reliability after one session of familiarization (Table 2). MIF ICCs improved from 
$\mathrm{r}=.58(p<.01)$ to $.86(p<.01)$, whereas $\mathrm{K}_{\text {ank }}$ ICCs improved in a similar manner from $r=.43(p<.05)$ to $r=.93(p<.01)$. This is supported by concurrent increases in MIF and $\mathrm{K}_{\mathrm{ank}}$ values. MIF increased by $7.9 \%$ from Days 1 to $2(p<.05)$ and by $2.8 \%$ from Days 2 to 3, with $\mathrm{K}_{\text {ank }}$ data increasing in a similar pattern by $12.2 \%(p<.01)$ and $2.8 \%(p<.05)$ between Days 1 and 2 and Days 2 and 3 respectively. Even though a familiarization increased the reliability of such measures, $\mathrm{K}_{\mathrm{ank}}$ strength and significance increases suggest additional testing sessions of this measure may be warranted.

The current $\mathrm{K}_{\text {ank }}$ findings (Table 1) appear to be marginally higher than maximum $\mathrm{K}_{\text {ank }}$ values that have been previously reported (Hunter \& Spriggs, 2000; McNair \& Stanley, 1996; Murphy et al., 2003; Watsford et al., 2003). However, such comparisons must be considered in light of the potential variations in the training state of subjects and methodological procedures used to determine $\mathrm{K}_{\text {ank }}$. Previous $\mathrm{K}_{\mathrm{ank}}$ research using active male subjects (Murphy et al. 2003) did not incorporate any familiarization session but reported reliability coefficients of between .89 and $.95(p<.01)$. Indeed, the execution of an isometric contraction is more common in trained and male subjects compared with the relatively untrained female subjects used in the current investigation. Other research has also shown that a learning effect is present for isometric force testing of relatively inexperienced subjects, with multiple trials required before a reliable result can be established (Bemben et al., 1992; Meldrum et al., 2003). This finding was confirmed in the variation of our MIF data between days (Table 1) and the higher reliability scores achieved after one session of testing (Table 2). Therefore it appears that the female subjects used in the current study are still potentially vulnerable to variations in MIF capacities, thus affecting the reliability of $\mathrm{K}_{\mathrm{ank}}$ results.

In terms of methodology comparisons, a bilateral $\mathrm{K}_{\text {ank }}$ assessment protocol was used in this study, whereas comparative studies have measured $\mathrm{K}_{\text {ank }}$ unilaterally (Hunter \& Spriggs, 2000; McNair \& Stanley, 1996; Murphy et al., 2003; Spurrs et al., 2003). Other studies have also used loadings above (Hunter \& Spriggs, 2000; Murphy et al., 2003; Spurrs et al., 2003) and below (Hunter \& Spriggs, 2000; McNair \& Stanley, 1996, Spurrs et al. 2003) the amount of $70 \%$ of MIF used in the current investigation. The MIF result directly determines the loading to be used for the oscillation test; thus, higher applied loadings will result in higher $\mathrm{K}_{\mathrm{ank}}$ values, with those approaching $70 \%$ of MIF causing stiffness levels to plateau (Walshe et al. 1996) as the series elastic component of the muscle reaches the same level of the tendon in the complex (Shorten 1987). This negative exponential load-stiffness trend could therefore be responsible for a large part of the variation between the current results and previously reported values. Since the oscillation test procedure requires a MIF test to be conducted beforehand, it is not clear whether the differences in $\mathrm{K}_{\text {ank }}$ measurements are due to a lack of test familiarization or because the MIF values were incorrect for this group of subjects in the early test sessions. Regardless, these findings highlight the need for the development of a standard protocol for $\mathrm{K}_{\mathrm{ank}}$ testing that includes at least one familiarization session so that accurate and reliable comparisons can be made within population groups.

The absence of significant relationships between $\mathrm{K}_{\text {leg }}$ and $\mathrm{K}_{\text {ank }}$ (Table 3 ) in the current study suggests that $\mathrm{K}_{\mathrm{ank}}$ may not potentially be a primary mechanism for modulating $\mathrm{K}_{\mathrm{leg}}$ at bounding movement frequencies of $2.2 \mathrm{~Hz}$ and $3.2 \mathrm{~Hz}$. Previous research has concluded $\mathrm{K}_{\mathrm{ank}}$ modulation to be a principal strategy for $\mathrm{K}_{\mathrm{leg}}$ adjustment when hopping under normal or maximum height conditions (Farley \& Morgenroth, 1999) or when hopping at preferred frequency $(2.2$ $\mathrm{Hz}$ ) on surfaces of varying compliance (Farley et al., 1998). Given the context of the current findings and that previous authors have found only minor hip joint influence on $\mathrm{K}_{\mathrm{leg}}$ (Farley et al., 1998), knee joint stiffness could possibly be an underestimated $\mathrm{K}_{\text {leg }}$ adjustment mechanism for the cyclic bounding frequencies tested in the current study. Derrick et al. (1998) reported that the knee joint assumes greater importance on energy absorption compared to the ankle joint when analyzing stride length in running actions, whereas Arampatzis et al. (1999) found that $\mathrm{K}_{\text {leg }}$ during running submaximally up to $6.5 \mathrm{~m} \cdot \mathrm{s}^{-1}$ was primarily influenced by changes in knee stiffness rather than $\mathrm{K}_{\text {ank }}$. It is therefore speculated that the musculoskeletal loadings of the cyclical action utilized could influence not only the overall level of $\mathrm{K}_{\text {leg }}$ but potentially the relative contribution of $\mathrm{K}_{\mathrm{ank}}$ and knee stiffness. At lower $\mathrm{K}_{\mathrm{leg}}$ levels, the knee may assume greater modulative responsibility, whereas $\mathrm{K}_{\text {ank }}$ may play a more significant role during faster leg actions such as running. Considering that this 
postulation is at best inconclusive and that $\mathrm{K}_{\mathrm{leg}}$ and $\mathrm{K}_{\text {ank }}$ in this study, were measured in functional and isolated contexts, respectively, further examination of individual joint stiffness contribution to $\mathrm{K}_{\mathrm{leg}}$ is recommended for future research.

Based on the results of the current study, it can be concluded that a strictly controlled $\mathrm{K}_{\mathrm{leg}}$ assessment protocol produces very reliable results in female subjects. It is suggested that future $\mathrm{K}_{\operatorname{leg}}$ assessment research should incorporate methodological strategies similar to those used in the current study. Furthermore, the current study has demonstrated that when assessing $\mathrm{K}_{\text {ank }}$ in relatively untrained female subjects using the oscillation method, at least one, and possibly more $\mathrm{K}_{\mathrm{ank}}$ familiarization sessions are needed to improve test reliability. Caution should be shown when interpreting findings from $\mathrm{K}_{\mathrm{ank}}$ research that has not incorporated any type of familiarization, particularly when untrained subjects are involved. Future research should examine the influences of familiarization on a wider range of subject groups to see whether the same findings are apparent. Finally, $\mathrm{K}_{\mathrm{ank}}$ does not appear to be a primary modulator of $\mathrm{K}_{\text {leg }}$ based on current procedures used to assess both measures. The absence of a relationship between $\mathrm{K}_{\mathrm{leg}}$ and $\mathrm{K}_{\mathrm{ank}}$ is potentially explained by the overall level of $\mathrm{K}_{\mathrm{leg}}$ and the discrepancy in knee joint angles between the two test procedures. The future development of an experimental model assessing both $\mathrm{K}_{\mathrm{leg}}$ and $\mathrm{K}_{\mathrm{ank}}$ in similar experimental contexts is therefore suggested.

\section{References}

Arampatzis, A., Bruggemann, G.P., \& Metzler, V. (1999). The effect of speed on leg stiffness and joint kinetics in human running. Journal of Biomechanics, 32, 1349-1353.

Asmussen, E., \& Bonde-Petersen, F. (1974). Storage of elastic energy in skeletal muscles in man. Acta Physiologica Scandinavia, 91, 385-392.

Bemben, M.G., Massey, B.H., Boileau, R.A., \& Misner, J.E. (1992). Reliability of isometric force-time curve parameters for men aged 20 to 79 years. Journal of Applied Sports Science Research, 6, 158-164.

Blickhan, R. (1989). The spring-mass model for running and hopping. Journal of Biomechanics, 22, 1217-1227.

Bobbert, M.F., Gerritsen, K.G., Litjens, M.C., \& Van Soest, A.J. (1996). Why is countermovement jump height greater than squat jump height? Medicine and Science in Sports and Exercise, 28, 1402-1412.

Bret, C., Rahmani, A., Dufour, A.B., Messonnier, L., \& Lacour, J.R. (2002). Leg strength and stiffness as ability factors in $100 \mathrm{~m}$ sprint running. Journal of Sports Medicine and Physical Fitness, 42, 274-281.

Butler, R.J., Cowell III, H.P., \& Davis, I.M. (2003). Lower extremity stiffness: implications for performance and injury. Clinical Biomechanics, 18, 511-517.

Cavagna, G.A. (1975). Force platforms as ergometers. Journal of Applied Physiology, 39, 174-179.

Chelly, S.M., \& Denis, C. (2001). Leg power and hopping stiffness: Relationship with sprint running performance. Medicine and Science in Sports and Exercise, 33, 326-333.

Derrick, T.R., Hamill, J., \& Caldwell, G.E. (1998). Energy absorption of impacts during running at various stride lengths. Medicine and Science in Sports and Exercise, 20, 128-135.

Dietz, V., Schmidtbleicher, D., \& Noth, J. (1979). Neuronal mechanisms of human locomotion. Journal of Neurophysiology, 42, 1212-1222.

Farley, C.T., Blickhan, R., Saito, J., \& Taylor, C.R. (1991). Hopping frequency in humans: A test of how springs set stride frequency in bouncing gaits. Journal of Applied Physiology, 71, 2127-2132.

Farley, C.T., Houdijk, H.H., Van Strien, C., \& Louie, M. (1998). Mechanism of leg stiffness adjustment for hopping on surfaces of different stiffnesses. Journal of Applied Physiology, $85,1044-1055$.

Farley, C.T., \& Morgenroth, D.C. (1999). Leg stiffness primarily depends on ankle stiffness during human hopping. Journal of Biomechanics, 32, 267-273.

Ferris, D.P., \& Farley, C.T. (1997). Interaction of leg stiffness and surfaces stiffness during human hopping. Journal of Applied Physiology, 82, 15-22.

Gunther, M., \& Blickhan, R. (2002). Joint stiffness of the ankle and the knee in running. Journal of Biomechanics, 35, 1459-1474.

Hotelling, H. (1940). The selection of variates for use in prediction, with some comments on the general problem of nuisance parameters. The Annals of Mathematical Statistics, 11, 271-283.

Hunter, D.G., \& Spriggs, J. (2000). Investigation into the relationship between the passive flexibility and active stiffness of the ankle plantar-flexor muscles. Clinical Biomechanics, 15, 600-606.

Komi, P.V., \& Bosco, C. (1978). Utilization of stored elastic energy in leg extensor muscles by men and women. Medicine and Science in Sports and Exercise, 10, 261-265.

Kuitunen, S., Komi, P.V., \& Kyrolainen H. (2002). Knee and ankle joint stiffness in sprint running. Medicine and Science in Sports and Exercise, 34, 166-173.

McMahon, T.A., \& Cheng, G.C. (1990). The mechanics of running: How does stiffness couple with speed? Journal of Biomechanics, 23, Suppl. 1, 65-78.

McNair, P.J., \& Stanley, S.N. (1996). Effect of passive stretching and jogging on the series elastic muscle stiffness and range of motion of the ankle joint. British Journal of Sports Medicine, 30, 313-318.

Meldrum, D., Cahalane, E., Keogan, F., \& Hardiman, O. (2003). Maximum voluntary isometric contraction: investigation of reliability and learning effect. Amyotrophic Lateral Sclerosis and Other Motor Neuron Disorders, 4, 36-44. 
Murphy, A.J., Watsford, M.L., Coutts, A.J., \& Pine, M.J. (2003). Reliability of a test of musculotendinous stiffness. Physical Therapy in Sport, 4, 175-181.

Sale, D.G. (1991). Testing Strength and Power. In J.D. MacDougall, H.A. Wenger, \& H.J. Green (Eds.), Physiological Testing of the High-Performance Athlete (2nd ed.). Champaign, IL: Human Kinetics.

Shorten, M.R. (1987). Muscle elasticity and human performance. In B. Van Gheluwe \& J. Atha (Eds.), Medicine and Sport Science, Volume 25. Current Research in Sports Biomechanics. Basel, Switzerland: Karger.

Sinkjaer, T., Toft, E., Andreassen, S., \& Hornemann, B.C. (1988). Muscle stiffness in human ankle dorsiflexors: intrinsic and reflex components. Journal of Neurophysiology, 60, 1110-1121.

Spurrs, R.W., Murphy, A.J., \& Watsford, M.L(2003). The effect of plyometric training on distance running performance. European Journal of Applied Physiology, 89, 1-7.
Stefanyshyn, D.J., \& Nigg, B.M. (1988). Dynamic angular stiffness of the ankle joint during running and sprinting. Journal of Applied Biomechanics, 14, 292-299.

Walshe, A.D., \& Wilson, G.J. (1997). The influence of musculotendinous stiffness on drop jump performance. Canadian Journal of Applied Physiology, 22, 117-132.

Walshe, A.D., Wilson, G.J., \& Murphy, A.J. (1996). The validity and reliability of a test of lower body musculotendinous stiffness. European Journal of Applied Physiology, 73, 332-339.

Watsford, M.L., Murphy, A.J., Spinks, W.L., \& Walshe, A.D. (2003). Creatine supplementation and its effect on musculotendinous stiffness and performance. Journal of Strength and Conditioning Research, 17, 26-33.

Wilson, G.J., Elliott, B.C., \& Wood, G.A. (1992). Stretch shorten cycle performance enhancement through flexibility training. Medicine and Science in Sports and Exercise, 24, 116-123. 